\title{
VARIABILIDADE SAZONAL DA EVAPOTRANSPIRAÇÃO RELATIVA EM CAMPINAS (SP): CARACTERIZAÇÃO CLIMÁTICA E ANÁLISE DE TENDÊNCIAS $\left({ }^{1}\right)$
}

\author{
GABRIEL CONSTANTINO BLAIN $(2 *)$; MARY TOSHIE KAYANO $\left({ }^{3}\right)$; \\ PAULO CESAR SENTELHAS $\left({ }^{4}\right)$; JORGE LULU $\left({ }^{4}\right)$
}

\begin{abstract}
RESUMO
O conhecimento da probabilidade de ocorrência da razão entre a evapotranspiração real e a potencial (ETR/ETP ou evapotranspiração relativa), é fundamental para a determinação de épocas mais adequadas de plantio/semeadura, podendo constituir-se em importante etapa para o zoneamento de riscos climáticos. Por meio da função densidade de probabilidade beta, determinou-se a probabilidade de ocorrência de valores de ETR/ETP na região de Campinas (SP), com base no período entre 1890 ae2006. A mesma análise foi realizada para os períodos de 1890 a 1918, 1919 a 1947, 1948 a 1976 (vinte e nove anos) e 1977 a 2006 (trinta anos), a fim de investigar a variabilidade das probabilidades de ocorrência desse parâmetro. As análises permitiram inferir que: a) em janeiro são observadas as maiores probabilidades de ocorrência de valores de ETR/ETP $=1 ; b$ ) sob o ponto de vista de reposição das perdas por evapotranspiração, os períodos mais críticos do calendário agrícola local ocorrem em abril, outubro e no primeiro decêndio de novembro; e c) método empregado não nos permite detectar significativas alterações na freqüência temporal dos valores de ETR/ETP durante o período analisado.
\end{abstract}

Palavras-chave: zoneamento de riscos climáticos, ETR/ETP, distribuição beta.

\section{ABSTRACT \\ SEASONAL VARIABILITY OF RELATIVE EVAPOTRANSPIRATION IN CAMPINAS (SP): CLIMATIC CHARACTERIZATION AND TRENDS ANALYSIS}

The knowledge of the probability of occurrence of the ratio between the actual evapotranspiration and the potential evapotranspiration (ETR/ETP) is fundamental to choose the best sowing/plantation seasons, and might be important to the climatic risks zoning. Using the beta probability density function it was determined the probability of occurrence of the ETR/ETP values in the region of Campinas, São Paulo State, Brazil for the period from 1890 to 2006. The same analysis was carried out considering the years from 1890 to 1918, 1919 to 1947, 1948 to 1976 (twenty-nine years) and 1977 to 2006 (thirty years), in order to investigate the variability of the ETR/ETP probabilities of occurrence. The analyses allowed to infer that: a) The ETR/ $\mathrm{ETP}=1$ cases have the largest probability of occurrence in January; $b$ ) under the point of view of the replacement of evapotranspiration losses, the most critical periods for the local agricultural calendar are in April, October and the first 10-day interval of November; and c) The method employed does not allow us to detect significant alterations in the frequency of ETR/ETP values during the analyzed period.

Key words: climatic risks zoning, ETR/ETP, beta distribution.

$\left({ }^{1}\right)$ Recebido para publicação em 20 de fevereiro de 2008 e aceito em 6 de fevereiro de 2009.

$\left({ }^{2}\right)$ Centro de Ecofisiologia e Biofísica, Instituto Agronômico, Caixa Postal 28, 13001-970 Campinas ( SP). E-mail: gabriel@iac.sp.gov.br $\left(^{*}\right)$ Autor correspondente.

$\left({ }^{3}\right)$ Instituto Nacional de Pesquisas Espaciais, Centro de Previsão de Tempo e Estudos Climáticos, Avenida dos Astronautas 1758, São José dos Campos (SP), Brasil.

$\left({ }^{4}\right)$ Escola Superior de Agricultura “Luiz de Queiroz" /USP, Departamento de Ciências Exatas, Piracicaba (SP), Brasil. 


\section{INTRODUÇÃO}

Uma das etapas fundamentais em estudos relativos ao zoneamento de riscos climáticos é a definição de épocas de plantio/semeadura/poda que proporcionem adequado grau de suprimento hídrico nos diferentes estádios fenológicos das culturas. A determinação de tais épocas pode, por sua vez, ser conceituada como conseqüência natural da correta caracterização climática, no que diz respeito ao entendimento da dinâmica das diversas sucessões dos tipos de tempo que ocorrem em uma região.

Sob este aspecto, o estudo probabilístico da variabilidade temporal de variáveis ambientais, ligadas à reposição das perdas por evapotranspiração de um cultivo, constitui-se em um importante passo no desenvolvimento da agricultura sustentável. Assim, torna-se importante investigar quais parâmetros podem ser utilizados para avaliar o potencial de atendimento hídrico às culturas e como o conhecimento das probabilidades de ocorrência desse potencial (incluindo suas limitações) contribui para estudos dessa natureza.

A evapotranspiração real (ETR) pode ser definida como a quantidade de água utilizada por uma cultura padrão em uma superfície padrão em dado período. Limitada apenas pelo balanço vertical de energia, a evapotranspiração potencial (ETP) pode, por sua vez, ser conceituada como a energia disponível no meio capaz de impor determinada demanda evapotranspirativa a essa cultura padrão. Com isso, nota-se que valores da razão ETR/ETP (evapotranspiração relativa) inferiores a 1 indicam que o sistema solo-planta não foi capaz de suprir a demanda evapotranspirativa imposta pela atmosfera em dado período. O fechamento estomático e a queda da produtividade agrícola são conseqüências naturais do fato acima descrito.

Diversos trabalhos correlacionam os valores da ETR/ETP ao rendimento/produtividade agrícola - Moraes et al. (1998) desenvolveram um modelo de quebra de rendimento da soja na região de Ribeirão Preto (SP); ORTOLANI et al. (1998) desenvolveram um modelo de estimativa de produtividade da seringueira; CAMARGO e HUBBARD (1999) desenvolveram um índice de seca agrícola para a cultura do sorgo; Piccini et al. (1999) desenvolveram um modelo de estimativa de quebra de rendimento da cultura do café e o modelo FAO apresentado por Doorenbos e KASSAM (1994), são exemplos dessa correlação. Esses autores também demonstram a importância do conhecimento da variabilidade temporal dessa variável agrometeorológica na determinação de épocas mais adequadas de plantio/semeadura/podas. Esse conhecimento pode constituir-se em importante etapa para o zoneamento de riscos climáticos.
É interessante ressaltar que o fato da ETR/ ETP possuir intervalos inferiores e superiores conhecidos $[0,1]$ e ser largamente utilizada em modelos de produtividade agrícola, torna o uso dessa relação mais vantajoso em relação a outros parâmetros do balanço hídrico climatológico.

Entretanto, em sua essência, a probabilidade é o método que permite ao pesquisador usar informações de uma amostra de dados, para fazer inferências ou descrever uma população desconhecida da qual a amostra foi obtida. Portanto, torna-se evidente que uma das maiores dificuldades em análises do clima e, em especial, no estudo da variabilidade temporal da ETR/ETP é determinar se a amostra (número de dados disponíveis) é suficiente para descrever a população (caracterização climática) da qual a amostra foi extraída.

Do ponto de vista meteorológico, essa dificuldade é relatada por Ріттоск et al. (1978) ao afirmarem que o fluxo de energia solar incidente sobre o globo terrestre interage com a atmosfera, oceanos, criosfera e biosfera de várias maneiras, tais como armazenamento, re-emissão ou redistribuição das ondas eletromagnéticas. As manifestações dinâmicas ou termodinâmicas causadas por esses processos naturais podem ser avaliadas instantaneamente (tempo) ou em um período mais longo (clima). Não há, entretanto, divisão clara entre quando termina o estudo do tempo e começa o do clima. Neste aspecto, a Organização Meteorológica Mundial indica que o intervalo de tempo mínimo necessário para a caracterização climática de uma região é de trinta anos.

Aliada a essa dificuldade, afirmações como as do IPCC (2001 e 2007), que indicam possíveis mudanças na variabilidade climática dos eventos meteorológicos, fazem com que a caracterização estatística de séries temporais de dados climáticos deva considerar a existência de possíveis alterações no regime esperado das variáveis em estudo.

Analisando possíveis alterações na distribuição temporal dos valores de ETR/ETP, entre os anos de 1890 e 2005 na região de Campinas, BlaiN et al. (2007) indicaram, considerando a análise dos parâmetros da distribuição beta, não haver indícios de significativas alterações de ordem climática na freqüência temporal dos valores dessa variável agrometeorológica. Entretanto, esses autores relataram também que no período de 1977 a 2005, houve intensificação das condições de deficiência hídrica no mês de agosto e queda da densidade de probabilidade de valores de ETR/ETP maiores que 0,8 no primeiro decêndio de novembro. 
Assim, o objetivo do trabalho é determinar a probabilidade de ocorrência de valores da evapotranspiração relativa na região de Campinas (SP), com base no período entre 1890 e 2006 . A mesma análise foi realizada para os períodos de 1890 a 1918, 1919 a 1947, 1948 a 1976 (vinte e nove anos) e 1977 a 2006 (trinta anos), a fim de investigar a variabilidade das probabilidades de ocorrência da ETR/ETP nos cento e dezessete anos de observações meteorológicas em Campinas (SP).

\section{MATERIAL E MÉTODOS}

Foram utilizados dados diários de precipitação pluvial e de temperatura do ar do posto meteorológico do município de Campinas, SP $\left(22^{\circ} 54^{\prime} \mathrm{S} ; 47^{\circ} 05^{\prime} \mathrm{W}\right.$; $669 \mathrm{~m}$ ) pertencente ao Instituto Agronômico da Secretaria de Agricultura e Abastecimento do Estado de São Paulo. De acordo com a classificação de Thornthwaite, o clima desse município é B1rB'4a (Pereira et al., 2002). Essa sigla indica um clima úmido, sem ou com pouca deficiência hídrica, com evapotranspiração anual média entre 997 e 1140 mm, em que de 48 a $51,9 \%$ dessa ETP anual ocorre nos meses de verão. Nessa região atuam tanto sistemas tropicais como polares conforme descrito em MonTEIRO (1973).

O modelo do Balanço hídrico climatológico de THORNTHWAITE e MATHER (1955) foi usado para estimar os valores decendiais da ETR considerando-se o valor inicial da capacidade de água disponível no solo (CAD) igual a $100 \mathrm{~mm}$. Os valores da ETP, também na escala decendial, foram calculados conforme o modelo proposto por THORNTHWAITE (1948).

A função densidade de probabilidade beta foi utilizada na estimação da probabilidade de ocorrência dos valores da razão ETR/ETP para os períodos 1890 a 2006, 1890 a 1918, 1919 a 1947, 1948 a 1976 e 1977 a 2006. A fim de verificar a influência da variação na CAD na variabilidade amostral da probabilidade de ocorrência associada aos valores de ETR, os cálculos acima descritos foram re-feitos considerando os valores de CAD 25 e $150 \mathrm{~mm}$. De acordo com PeREIRA et al. (2002) os três valores de CAD representam espécies hortícolas $(25 \mathrm{~mm})$; culturas anuais e perenes (100 mm) e espécies florestais (150 mm).

Segundo WILKS (2006), para uma variável x, a função densidade de probabilidade beta é:

$$
f(x)=\left[\frac{\Gamma(p+q)}{\Gamma(p)+\Gamma(q)}\right] x^{(p-1)}(1-x)^{(q-1)} \quad 0 \leq \mathrm{x} \leq 1 \quad \mathrm{p}, \mathrm{q}>0
$$

Em que,

p e q são os parâmetros da distribuição beta estimados pelo método dos momentos.
BLAin et al. (2007), por meio do teste de Kolmogorov-Smirnov, afirmaram que a distribuição empírica sob investigação é proveniente de uma população com distribuição beta.

\section{RESULTADOS E DISCUSSÃO}

Sob o ponto de vista do padrão climático da região de Campinas, observa-se, por meio da Tabela 1 e da Figura1, que entre abril e agosto, o número de casos em que o valor da ETR iguala-se ao da ETP tende a declinar. Esse período seco tem seu auge em agosto, quando as menores probabilidades de ocorrência são observadas para os maiores valores de ETR/ETP. Após esse mês, a freqüência de valores de ETR/ETP =1 começa a aumentar, atingindo seu valor máximo em janeiro (mês com menor risco de seca agrícola).

É interessante ressaltar a queda na probabilidade de ocorrência dos valores de ETR/ETP = 0,8 registrada em abril, em especial no último decêndio deste mês, e sua posterior elevação durante o mês de maio. A despeito das possíveis forçantes físicas que resultam nestes níveis de probabilidade, este quadro indica que o mês de abril pode ser considerado (tendo em vista o calendário agrícola local) um dos meses mais críticos para a agricultura, no que diz respeito à reposição necessária das perdas por evapotranspiração das culturas na região de Campinas (SP).

Semelhante a abril, porém com menor intensidade, outubro e início de novembro podem ser também considerados críticos para as culturas que, nessas épocas, estejam em estádios fenológicos muito sensíveis ao déficit hídrico. Sob o enfoque agrícola, a partir do segundo decêndio de novembro, a estação úmida, aqui conceituada como a estação em que os valores de ETR igualam-se aos da ETP, tem $80 \%$ de possibilidade de estar estabelecida, sendo, portanto, desejável que a fase reprodutiva do calendário agrícola de Campinas ocorra a partir desse período.

Como exemplo, pode ser utilizado o esquema das seis fases fenológicas do cafeeiro arábica, desenvolvido por CAMARgo e CAMARgo (2001), nas condições climáticas tropicais do Brasil, apresentado na figura 2.

Considerando que o cafeeiro, para vegetar e frutificar normalmente necessita de umidade facilmente disponível no solo durante todo o período vegetativo e de frutificação, nota-se, comparando-se a Figura 1 à Figura 2, que em Campinas, o início do período reprodutivo do café arábica pode ser considerado crítico, pois com valores da evapotranspiração relativa iguais ou superiores a 0,8 há probabilidade de ocorrências superiores a $80 \%$ apenas após o primeiro decêndio de outubro. 
Tabela 1. Densidade de probabilidade das classes da relação entre a evapotranspiração real e evapotranspiração potencial (ETR/ETP), na escala decendial (Dec) considerando a capacidade de água disponível (CAD) igual a $100 \mathrm{~mm}$, de 1890 a 2006, na região de Campinas (SP), Brasil

\begin{tabular}{|c|c|c|c|c|c|c|c|}
\hline \multirow{2}{*}{ Mês } & \multicolumn{7}{|c|}{ ETR/ETP (1890 a 2006) } \\
\hline & Dec & 1 & $\geq 0,9$ & $\geq 0,8$ & $\geq 0,7$ & $\geq 0,6$ & $\geq 0,4$ \\
\hline \multirow[t]{3}{*}{ Janeiro } & 1 & 0,912 & 0,955 & 0,976 & 0,987 & 0,993 & 0.999 \\
\hline & 2 & 0,927 & 0,973 & 0,989 & 0,996 & 0,999 & 1.000 \\
\hline & 3 & 0,918 & 0,969 & 0,988 & 0,995 & 0,998 & 1.000 \\
\hline \multirow[t]{3}{*}{ Fevereiro } & 4 & 0,923 & 0,968 & 0,986 & 0,994 & 0,998 & 1.000 \\
\hline & 5 & 0,885 & 0,953 & 0,980 & 0,992 & 0,997 & 1.000 \\
\hline & 6 & 0,854 & 0,932 & 0,967 & 0,985 & 0,993 & 0.999 \\
\hline \multirow[t]{3}{*}{ Março } & 7 & 0,878 & 0,957 & 0,984 & 0,995 & 0,998 & 1.000 \\
\hline & 8 & 0,835 & 0,919 & 0,959 & 0,979 & 0,991 & 0.999 \\
\hline & 9 & 0,761 & 0,879 & 0,937 & 0,968 & 0,985 & 0.998 \\
\hline \multirow[t]{3}{*}{ Abril } & 10 & 0,692 & 0,834 & 0,908 & 0,951 & 0,976 & 0.996 \\
\hline & 11 & 0,548 & 0,746 & 0,860 & 0,927 & 0,965 & 0.995 \\
\hline & 12 & 0,402 & 0,599 & 0,735 & 0,831 & 0,899 & 0.975 \\
\hline \multirow[t]{3}{*}{ Maio } & 13 & 0,511 & 0,651 & 0,744 & 0,814 & 0,869 & 0.946 \\
\hline & 14 & 0,525 & 0,665 & 0,757 & 0,826 & 0,878 & 0.952 \\
\hline & 15 & 0,509 & 0,645 & 0,736 & 0,805 & 0,860 & 0.940 \\
\hline \multirow[t]{3}{*}{ Junho } & 16 & 0,507 & 0,655 & 0,753 & 0,826 & 0,881 & 0.956 \\
\hline & 17 & 0 , & 0,622 & 0,716 & 0,788 & 0,846 & 0.932 \\
\hline & 18 & 0,451 & 0,598 & 0,700 & 0,779 & 0,842 & 0.933 \\
\hline \multirow[t]{3}{*}{ Julho } & 19 & 0,396 & 0,550 & 0,661 & 0,748 & 0,818 & 0.923 \\
\hline & 20 & 0,333 & 0,491 & 0,610 & 0,706 & 0,786 & 0.908 \\
\hline & 21 & 0,239 & 0,394 & 0,522 & 0,632 & 0,727 & 0.878 \\
\hline \multirow[t]{3}{*}{ Agosto } & 22 & 0,275 & 0,425 & 0,545 & 0,646 & 0,733 & 0.874 \\
\hline & 23 & 0,206 & 0,350 & 0,473 & 0,582 & 0,679 & 0.844 \\
\hline & 24 & 0,271 & 0,410 & 0,522 & 0,617 & 0,701 & 0.844 \\
\hline \multirow[t]{3}{*}{ Setembro } & 25 & 0,355 & 0,479 & 0,572 & 0,650 & 0,718 & 0.837 \\
\hline & 26 & 0,420 & 0,533 & 0,614 & 0,680 & 0,738 & 0.841 \\
\hline & 27 & 0,527 & 0,623 & 0,688 & 0,741 & 0,786 & 0.866 \\
\hline \multirow[t]{3}{*}{ Outubro } & 28 & 0,569 & 0,671 & 0,739 & 0,793 & 0,837 & 0.910 \\
\hline & 29 & 0,710 & 0,786 & 0,835 & 0,871 & 0,901 & 0.947 \\
\hline & 30 & 0,713 & 0,803 & 0,858 & 0,897 & 0,927 & 0.969 \\
\hline \multirow[t]{3}{*}{ Novembro } & 31 & 0,695 & 0,801 & 0,865 & 0,908 & 0,940 & 0.979 \\
\hline & 32 & 0,857 & 0,906 & 0,935 & 0,954 & 0,968 & 0.987 \\
\hline & 33 & 0,806 & 0,887 & 0,931 & 0,958 & 0,976 & 0.994 \\
\hline \multirow[t]{3}{*}{ Dezembro } & 34 & 0,882 & 0,935 & 0,962 & 0,978 & 0,987 & 0.997 \\
\hline & 35 & 0,906 & 0,957 & 0,980 & 0,990 & 0,996 & 1.000 \\
\hline & 36 & 0,917 & 0,956 & 0,976 & 0,986 & 0,993 & 0.999 \\
\hline
\end{tabular}

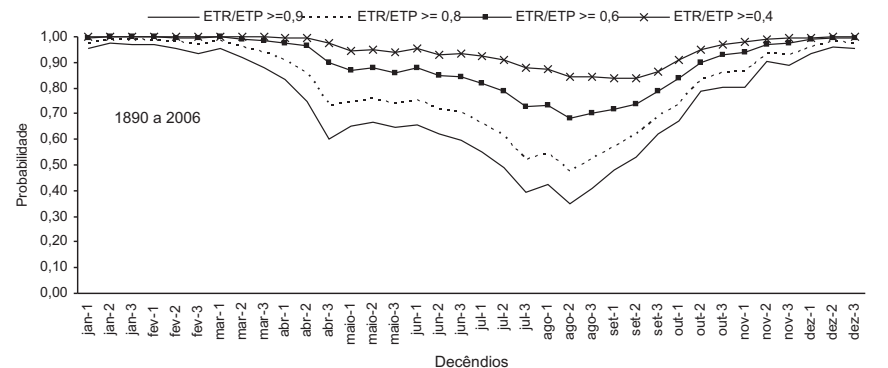

Figura 1. Densidade de probabilidade (DP) das classes da relação entre a evapotranspiração real e evapotranspiração potencial (ETR/ETP=0,9, ETR/ $\mathrm{ETP}=0,8, \mathrm{ETR} / \mathrm{ETP}=0,6$ e ETR/ETP=0,4), na escala decendial, considerando a capacidade de água disponível (CAD) igual a $100 \mathrm{~mm}$, na região de Campinas (SP), de 1890 a 2006.

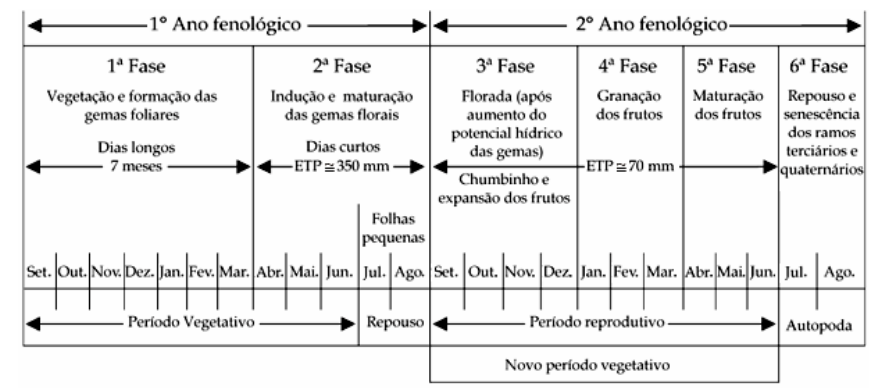

Figura 2. Esquema das seis fases fenológicas do cafeeiro arábica, nas condições climáticas tropicais do Brasil, durante 24 meses (CAMARgo e CAMARGo, 2001).

Sob o mesmo aspecto, a queda na probabilidade de ocorrência dos valores de ETR/ $\mathrm{ETP}=0,8$, registrada em abril, atesta que o início do período de indução e maturação das gemas florais, cujas exigências hídricas da cultura ainda são relativamente elevadas, é também uma fase bastante sujeita à quebra de produtividade causada pelo estresse hídrico na região em análise.

Em relação ao plantio de novas mudas, é interessante observar que no período entre o segundo decêndio de dezembro e o primeiro decêndio de fevereiro, a relação ETR/ETP=1 tem probabilidades de ocorrência superiores a $90 \%$ sendo, com isso, a fase do ano mais indicada para o plantio de novas plantas de café arábica.

São demonstradas nas Figuras 3, 4 e 5, as probabilidades de ocorrência das diferentes classes da relação ETR/ETP, na escala decendial, na região de Campinas, SP para os espaços amostrais de 1890 a 1918, 1919 a 1947, 1948 a 1976 e 1977 a 2006. 


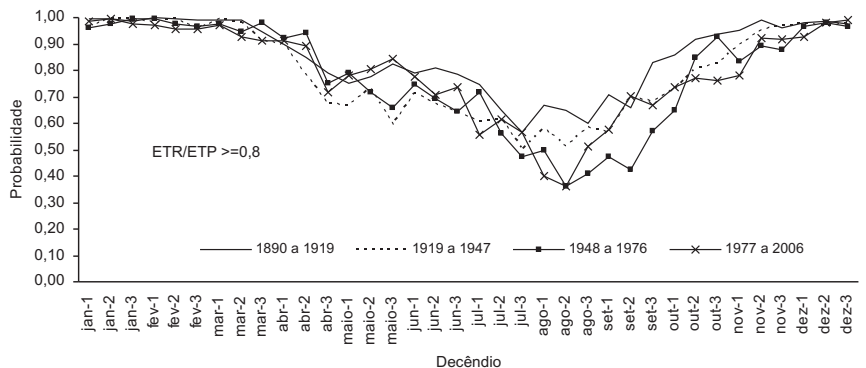

Figura 3. Probabilidade de ocorrência dos valores da relação entre a evapotranspiração real e potencial iguais ou superiores a 0,8 estimados com base nos períodos de 1890 a 1918, 1919 a 1947, 1948 a 1976 e 1977 a 2006 considerando a capacidade de água disponível (CAD) igual a $100 \mathrm{~mm}$, em Campinas (SP).

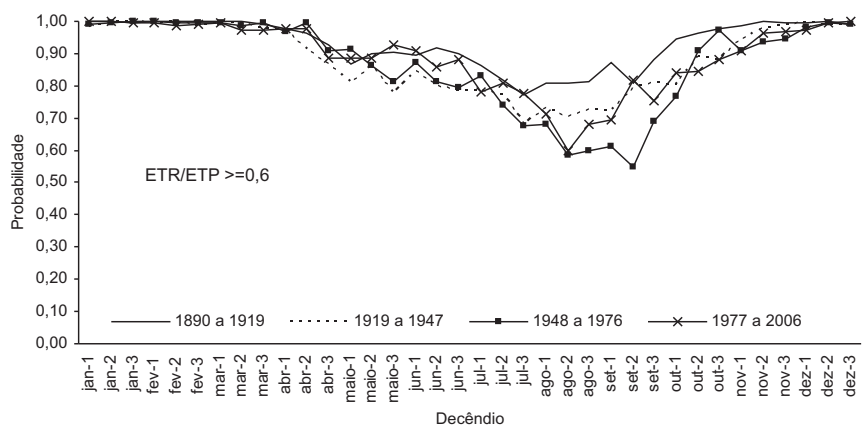

Figura 4. Probabilidade de ocorrência dos valores da relação entre a evapotranspiração real e potencial iguais ou superiores a 0,6 estimados com base nos períodos de 1890 a 1918, 1919 a 1947, 1948 a 1976 e 1977 a 2006 considerando a capacidade de água disponível (CAD) igual a $100 \mathrm{~mm}$, em Campinas (SP).

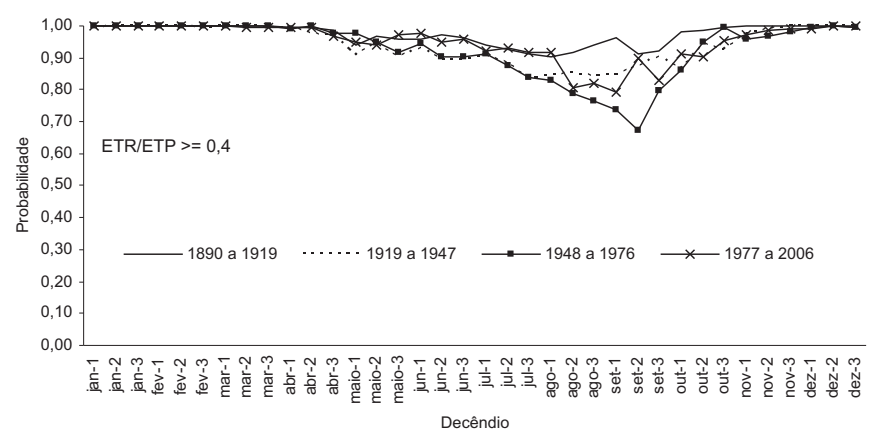

Figura 5. Probabilidade de ocorrência dos valores da relação entre a evapotranspiração real e potencial iguais ou superiores a 0,4 estimados com base nos períodos de 1890 a 1918, 1919 a 1947, 1948 a 1976 e 1977 a 2006, considerando a capacidade de água disponível (CAD) igual a $100 \mathrm{~mm}$, em Campinas (SP).

A análise das figuras 3 a 5 pode ser feita sob dois enfoques: a) Considerando as afirmações de PІтTOCK et al. (1978), de que possíveis alterações climáticas devem ser evidenciadas por meio de tendências contínuas na variabilidade de alguns elementos meteorológicos, verificar a existência dessas possíveis tendências na série em estudo para os períodos de 1890 a 1918, 1919 a 1947, 1948 a 1976 e 1977 a 2006; b) Verificar se as inferências realizadas em cada espaço amostral podem ser extrapoladas para os demais.

Essas duas abordagens são dependentes entre si, tendo em vista que a existência de tendências em uma série de dados pode impossibilitar que as descrições realizadas com base em um determinado período (amostra) sejam extrapoladas para outras épocas. Entretanto, seja qual for o enfoque adotado, deve-se também considerar a subjetividade na definição do termo clima e, consequentemente, nas definições dos conceitos de caracterização e alteração climática.

Ainda que extremamente precisa e corretamente embasada, a análise de dois períodos distintos (mesmo que longos e pertencentes ao mesmo local) não resultará em parâmetros estatísticos, relativos às duas épocas, exatamente iguais. Estabelecer um nível de significância que demonstre que tais diferenças são relativas a alterações de ordem climática, a deficiências do tamanho da série em estudo ou a variabilidade natural do clima da região é bastante difícil. No presente caso, deve-se também considerar que a região de Campinas situa-se em área de transição tropical/subtropical, com influências de sistemas tropicais e polares. Dessa forma, a conceituação dos termos clima, caracterização e alteração climática torna-se ainda mais subjetiva. Outro aspecto que deve ser considerado é a possível influência do clima urbano (ilhas de calor) nas leituras realizadas nas últimas décadas no posto meteorológico representativo do município de Campinas (SP). VICENTE e NUNES (2004) relataram que, como a maioria das cidades brasileiras, este município, um dos centros tecnológicos mais importantes do país, possui aproximadamente 1 milhão de habitantes. Nesse sentido, KAYANO e BlaIN (2007) indicaram a existência de tendências lineares (possivelmente resultante da urbanização) na série diária de temperatura mínima de Campinas (SP).

Adotando-se arbitrariamente o nível de probabilidade de ocorrência igual ou superior a $80 \%$ como o nível de segurança ( $20 \%$ de risco; Figura 3 ) para as atividades agrícolas, e assumindo que valores de $\mathrm{ETR} / \mathrm{ETP}=0,8$, constituem condições favoráveis ao desenvolvimento da maioria das culturas agrícolas da região, observa-se que nos períodos de 1890 a 1918, 1919 a 1947, 1948 a 1976 e 1977 a 2006 esse nível de probabilidade ocorre entre o terceiro decêndio de setembro e o segundo decêndio de abril; entre o segundo decêndio de outubro e primeiro decêndio de abril; entre o segundo decêndio de outubro e segundo decêndio de abril; entre o segundo decêndio de novembro e segundo decêndio de abril, respectivamente. 
Nota-se, portanto, atraso na retomada da estação favorável à reposição das perdas por evapotranspiração ocorrido nos últimos trinta anos da série. Considerandose os casos de ETR/ETP $=0,6$ e ETR/ETP $=0,4$, por meio das figuras 4 e 5 , verifica-se que entre o segundo decêndio de agosto e o primeiro decêndio de outubro, foram observadas as condições mais severas de deficiência hídrica no período entre 1948 e 1976.
No segundo e no terceiro decêndio de outubro, valores de ETR/ETP maiores que 0,4 tiveram as menores frequências de ocorrência entre 1977 e 2006.

Entretanto, em novembro, nessa classe crítica da razão ETR/ETP, notaram-se as densidades de probabilidades mais baixas entre 1948 e 1976.

Tabela 2. Decêndios e número de decêndios em cada espaço amostral com probabilidade de ocorrência igual ou superior à $80 \%$ para os valores de ETR/ETP = 0,8, ETR/ETP = 0,6 e ETR/ETP = 0,4, considerando a capacidade de água disponível (CAD) igual a $100 \mathrm{~mm}$, na região de Campinas (SP), Brasil

\begin{tabular}{lccccc}
\hline \multirow{2}{*}{ Razão } & \multicolumn{5}{c}{ Períodos (Espaços amostrais) } \\
\cline { 2 - 6 } & 1890 a 2006 & 1890 a 1918 & 1919 a 1947 & 1948 a 1976 & 1977 a 2006 \\
\hline ETR/ETP $=0,8$ & out-2 a abr-2 (19) & set-3 a abr-2 $(21)$ & out-2 a abr-1 (17) & out-2 a abr-2 (19) & nov-2 a abr-2 (16) \\
ETR/ETP =0,6 & out-1 a jul-1 (28) & ago-1 a jul-2 (35) & set-3 a jun-1 (23) & out-2 a jul-1 (25) & out-1 a ago-2 (31) \\
ETR $/$ ETP $=0,4$ & todo o período & todo o período & todo o período & out-1 a ago-1 (31) & set-2 a ago-3 (35) \\
\hline
\end{tabular}
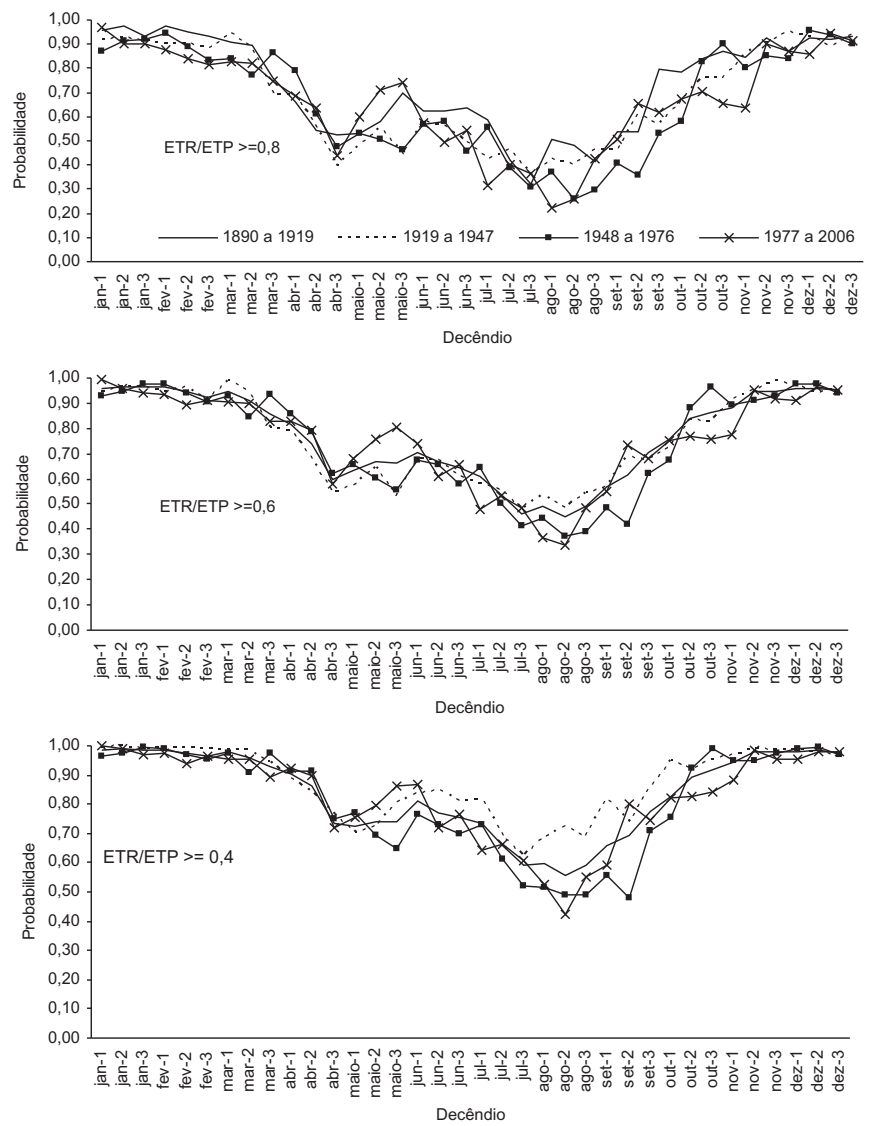

Figura 6. Probabilidade de ocorrência dos valores da relação entre a evapotranspiração real e potencial iguais ou superiores a 0,$8 ; 0,6$ e 0,4 estimados com base nos períodos de 1890 a 1918, 1919 a 1947, 1948 a 1976 e 1977 a 2006, considerando a capacidade de água disponível (CAD) igual a $25 \mathrm{~mm}$, em Campinas (SP).

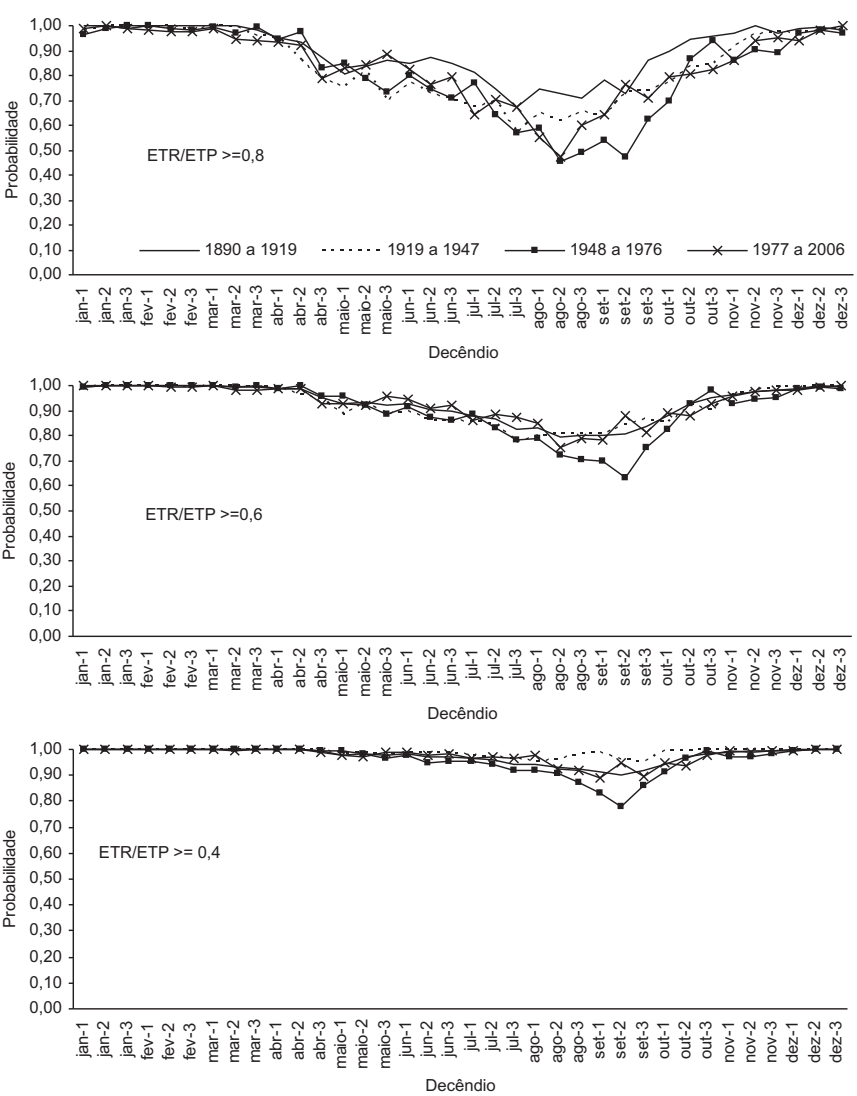

Figura 7. Probabilidade de ocorrência dos valores da relação entre a evapotranspiração real e potencial iguais ou superiores a 0,$8 ; 0,6$ e 0,4 estimados com base nos períodos de 1890 a 1918, 1919 a 1947, 1948 a 1976 e 1977 a 2006, considerando a capacidade de água disponível (CAD) igual a $150 \mathrm{~mm}$, em Campinas (SP). 


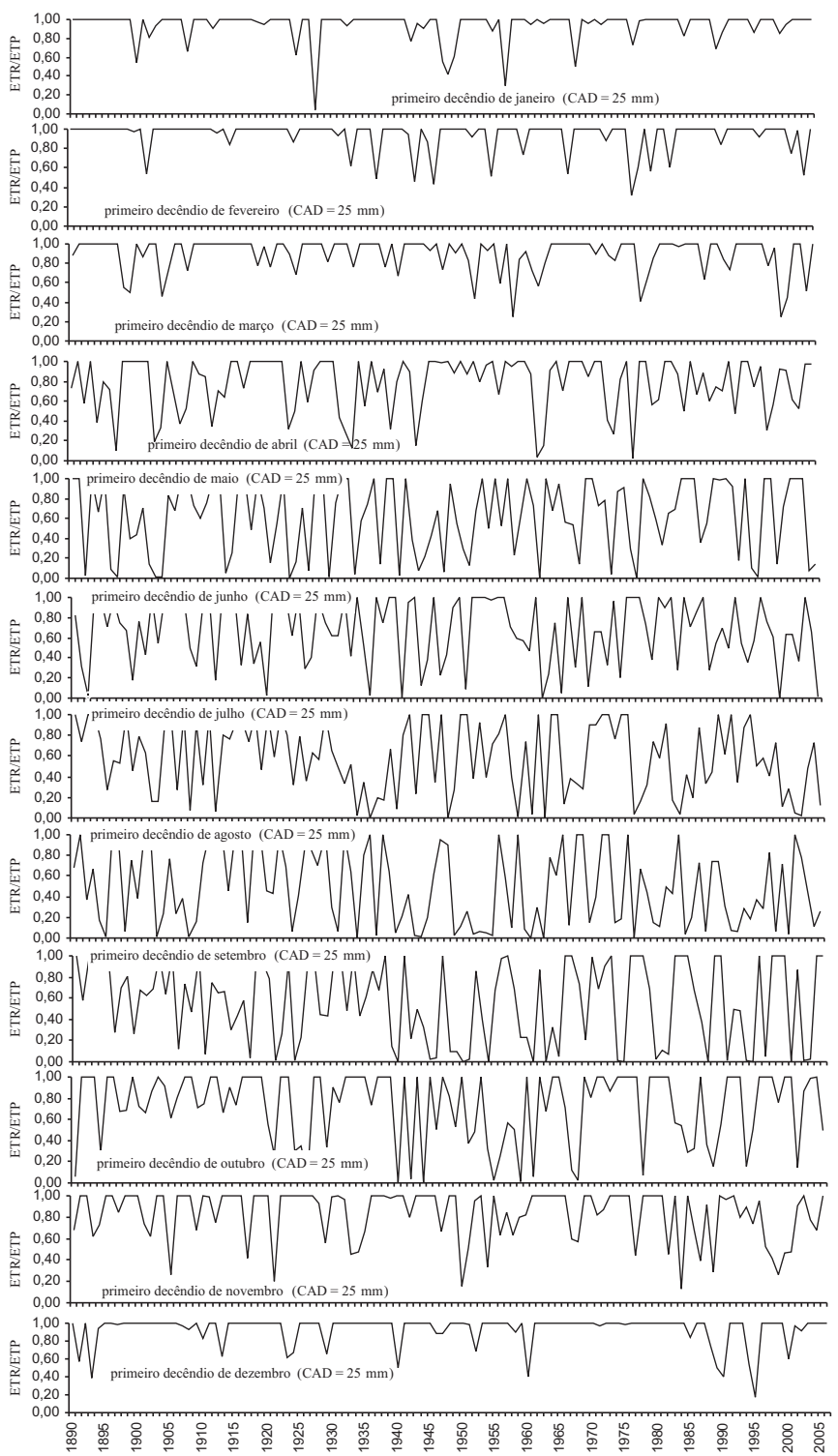

Figura 8. Séries temporais da evapotranspiração relativa. Capacidade de água disponível, de $25 \mathrm{~mm}$. Campinas (SP)

É apresentado na tabela 2 o número de decêndios em cada espaço amostral com probabilidade de ocorrência igual ou superior à $80 \%$ para os valores de ETR $/$ ETP $=0,8$, ETR $/$ ETP $=0,6$ e ETR $/ \mathrm{ETP}=0,4$

A comparação entre as figuras 3, 4 e 5 e a tabela 2 não evidencia tendências contínuas na variabilidade temporal da relação ETR/ETP entre os períodos de 1890 e 1918, 1919 e 1947, 1948 e 1976 e 1977 e 2006, não atendendo as afirmações de Ріттоск et al. (1978) quanto aos indícios de alterações climáticas.

Em relação à questão " $b$ " (verificar se as inferências realizadas em cada espaço amostral podem ser extrapoladas para os demais), uma resposta conservadora para o planejamento agrícola seria adotar os espaços amostrais com menor número de

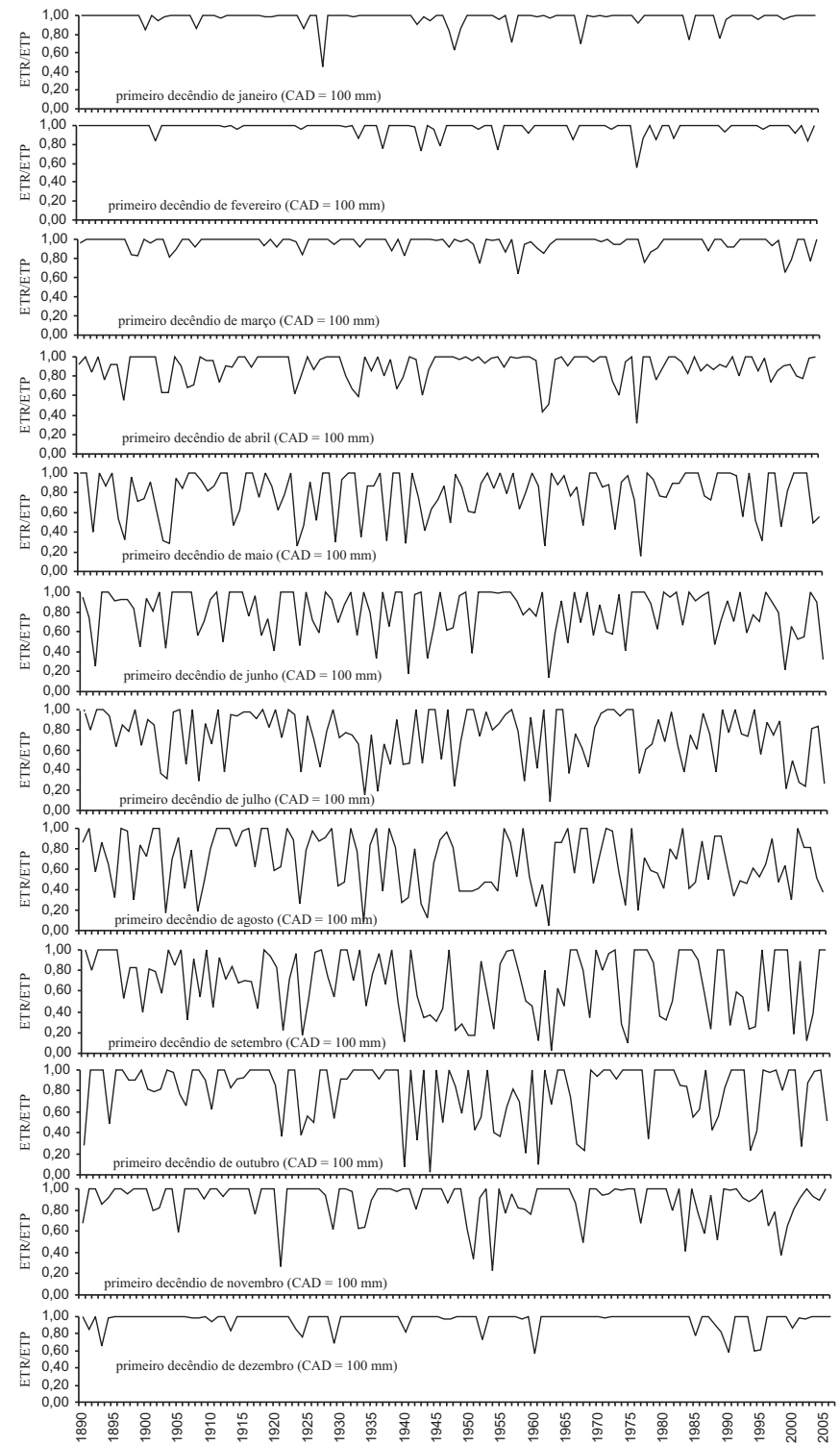

Figura 9. Séries temporais da evapotranspiração relativa.

Capacidade de água disponível, de $100 \mathrm{~mm}$. Campinas (SP).

decendios favoráveis a uma dada atividade, a fim de minimizar os riscos quanto ao estresse hídrico na região de Campinas (SP).

São demonstradas nas figuras 6 e 7 , as probabilidades de ocorrência das diferentes classes da relação ETR/ETP, na escala decendial, na região de Campinas (SP) para os espaços amostrais de 1890 a 1918, 1919 a 1947, 1948 a 1976 e 1977 a 2006 considerando-se os valores 25 e $150 \mathrm{~mm}$ de CAD.

Assim como observado para os casos de CAD $=100 \mathrm{~mm}$, a análise das figuras 6 e 7 não evidencia tendências contínuas na variabilidade temporal da relação ETR/ETP entre os períodos de 1890 a 1918, 1919 a 1947, 1948 a 1976 e 1977 a 2006, não atendendo, mais uma vez, as afirmações de Ріттоск et al. (1978) quanto aos indícios de alterações climáticas. 


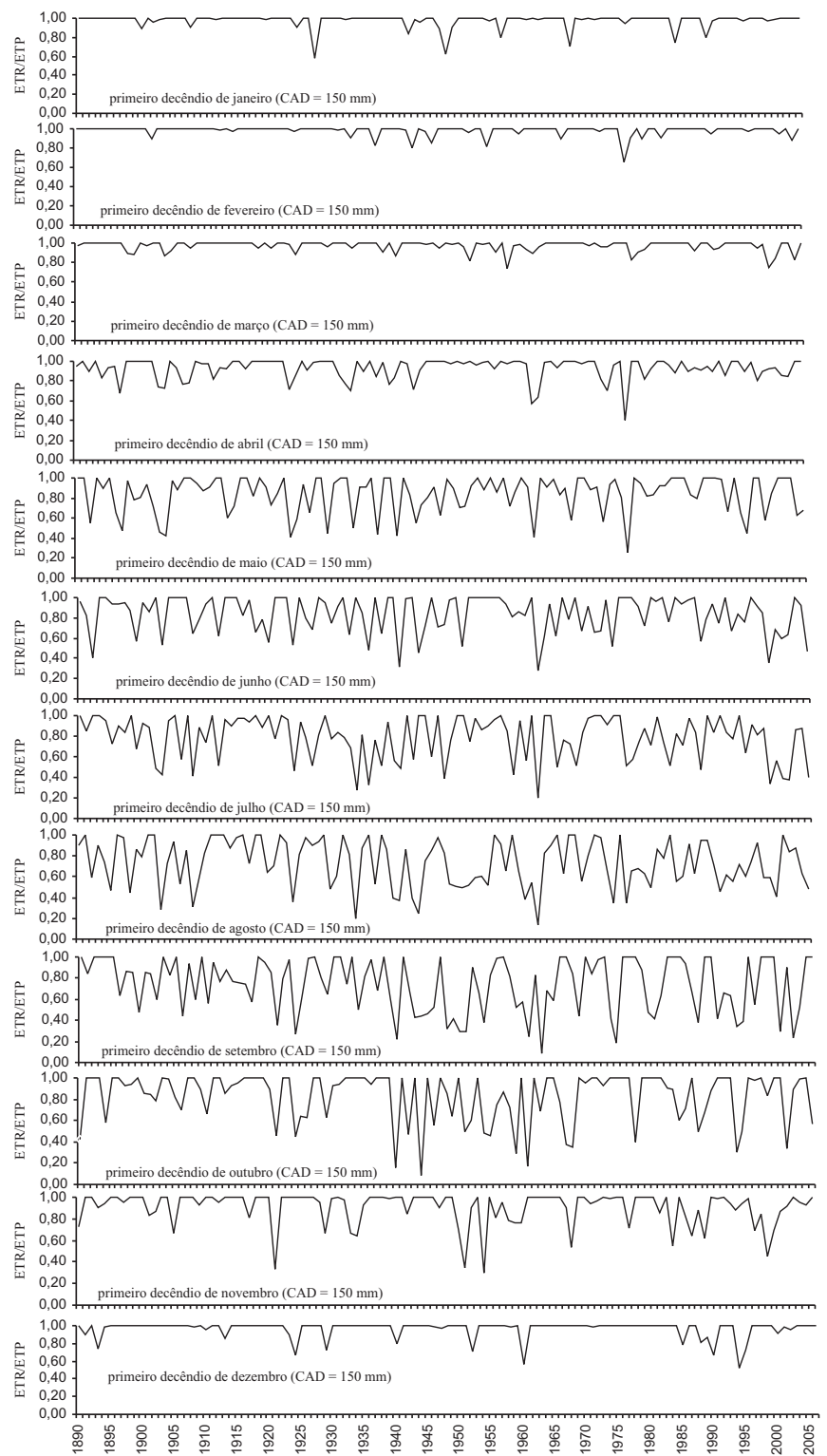

Figura 10. Séries temporais da evapotranspiração relativa. Capacidade de água disponível, de 150 mm, Campinas (SP)

Comparando-se as figuras 3, 6 e 7, verifica-se que o aumento da CAD aumenta o número de decêndios em que a relação ETR/ETP assume valores iguais ou superiores a 0,8 . Essa característica é conseqüente do fato de sistemas radiculares mais profundos serem menos sujeitas à ocorrência de secas agrícolas. Nesse aspecto, é importante ressaltar que o atraso na retomada da estação favorável à reposição das perdas por evapotranspiração ocorrido nos últimos trinta anos da série para CAD $=100 \mathrm{~mm}$ (Figura 3), foi também observado apenas para o valor de $\mathrm{CAD}=25 \mathrm{~mm}$. Esse atraso não ocorreu para os casos de $\mathrm{CAD}=150 \mathrm{~mm}$.
Dessa forma, considerando-se que trabalhos como os do Ipcc (2007) indicam possibilidade de aumento dos casos de seca agrícola, estudos de manejo agronômico e melhoramento genético, voltados ao aprofundamento do sistema radicular das espécies agrícolas, constituem-se em uma interessante alternativa para possível mitigação dos efeitos das prováveis mudanças climáticas apontadas pelo IPCC (2007).

A fim de ilustrar as características citadas no trabalho, nas figuras 8,9 e 10 são demonstradas as séries de ETR/ETP relativas aos primeiros decêndios de cada mês, para os valores 25, 100 e 150 mm de CAD.

Observando-se as figuras 8, 9 e 10, verifica-se que para o mesmo período, valores de CAD iguais a $25 \mathrm{~mm}$ tendem a ter valores mais baixos da relação ETR/ETP quando comparados aos observados, considerando-se CAD $=100 \mathrm{~mm}$ ou $C A D=150 \mathrm{~mm}$. Em outro aspecto, é mais uma vez evidente a inexistência de tendências climáticas contínuas nas séries decendiais de evapotranspiração relativa.

É interessante ressaltar que a Organização Mundial de Meteorologia define por meio da nota técnica número 79, OMM (1966), tendência climática como uma elevação (ou diminuição) suave e monótona nos valores médios de uma série meteorológica. Esse termo não é, necessariamente, restrito a tendências lineares em função do tempo, mas deve conter apenas um máximo, ou mínimo, no ponto final da série. Essa característica não é observada em nenhum dos casos apresentados nas figuras 8, 9 e 10 .

\section{CONCLUSÕES}

1. Em janeiro são observadas as maiores probabilidades de ocorrência de valores de ETR/ETP $=1$.

2. Abril, outubro e o primeiro decêndio de novembro podem, sob o ponto de vista de reposição das perdas por evapotranspiração, ser considerados os períodos mais críticos do calendário agrícola local.

3. Com base nos períodos de 1890 a 1918, 1919 a 1947, 1948 a 1976 e 1977 a 2006 não foi possível detectar-se indícios significativos de alterações de ordem climática na freqüência temporal dos valores decendiais de ETR/ETP, por meio do método empregado. Entretanto, para os valores de CAD iguais a 25 e $100 \mathrm{~mm}$, deve-se ressaltar o atraso na retomada da estação favorável à reposição das perdas por evapotranspiração ocorrido nos últimos trinta anos da série considerada. Esse atraso não ocorreu para CAD igual a $150 \mathrm{~mm}$. 


\section{REFERÊNCIAS}

BLAIN, G.C.; CAMARGO, M.B.P.; PEREIRA, A.R. Detection of possible alterations (trends) in the ten day period ratio between actual and potential evapotranspiration during the years 1890 to 2005 in the region of Campinas-SP-Brazil. In: CONGRESSO BRASILEIRO DE AGROMETEOROLOGIA, 15., 2007, Aracaju. Anais... Aracaju: Sociedade Brasileira de Agrometeorologia, 2007. (CD-ROM)

CAMARGO, A.P; CAMARGO, M.B.P. Definição e esquematização das fases fenológicas do cafeeiro arábica nas condições tropicais do Brasil. Bragantia, Campinas, v.60, n.1, p.65-68, 2001.

CAMARGO, M.B.P.; HUBBARD, K.G. Drought sensitivity indices for a sorghum crop. Journal of Production Agriculture, Madison, v.12, n.2, p.312-316, 1999.

DOORENBOS, J.; KASSAM, A.H. Efeito da água no rendimento das culturas. Campina Grande: UFPB, 1994. 306p.

KAYANO, M.T.; BLAIN, G.C. Análisis de ondeletas de series mensuales de precipitación, temperaturas máximas y mínimas mensuales en Campinas. In: CONGRESSO VENEZOLANO DE AGROMETEOROLOGIA, 4., 2007, Maracay. Anais... Maracay: Fundacite Táchira, 2007. (CD-ROM)

IPCC. Impacts, Adaptation and Vulnerability. Contribution of Working Group 2 to the Third Assessment Report of the Intergovernmental Panel on Climate Change. In: HOUGHTON, J.T. (Ed.). Climate Change. Cambridge: Cambridge University Press, 2001. 20p.

IPCC. The Physical Science Basis. Contribution of Working Group 1 to the Fourth Assessment Report of the Intergovernmental Panel on Climate Change. In: HOUGHTON, J.T. (Ed.). Climate Change. Cambridge: Cambridge University Press, 2007. 17p.

MONTEIRO, C.A.F. A dinâmica climática e as chuvas no estado de São Paulo: estudo geográfico sob forma de atlas. São Paulo: Universidade de São Paulo, Instituto de Geografia, 1973. 154p.

MORAES, A.V.C.; CAMARGO, M.B.P.; MASCARENHAS, H.A.A.; MIRANDA, M.A.C.; PEREIRA, J.C.V.A. Teste e análise de modelos agrometeorológicos de estimative de produtividade para a cultura da soja na região de Ribeirão Preto, SP. Bragantia, Campinas, v.57, n.2, p.393-406, 1998.

ORGANIZAÇÃO METEOROLÓGICA MUNDIAL. Climatic Change. Geneva, Switzerland: WMO, 1966. 80p.

ORTOLANI, A.A.; SENTELHAS, P.C.; CAMARGO, M.B.P.; PEZZOPANE, J.E.M.; GONÇALVES, P.S. Agrometeorological model for a seasonal ruber tree yield. Indian Journal of Natural Rubber Research, New Deli, v.11, n.1, p.8-14, 1998.

PEREIRA, A.R.; ANGELOCCI, L.R.; SENTELHAS, P.C. Agrometeorologia: fundamentos e aplicações práticas. Guaíba: Agropecuária, 2002. 479p.
PICCINI, A.G.; CAMARGO, M.B.P.; ORTOLANI, A.A.; FAZUOLI, L.C.; GALLO, P.B. Desenvolvimento e teste de modelos agrometeorologicos para a estimativa de produtividade do cafeeiro. Bragantia, Campinas, v.58, n.1, p.171-178, 1999.

PITTOCK, A.B.; FRAKES, L.A.; JESSEN, D.; PETERSON, J.A.; ZILLMAN, J.W. Climatic change and variability: a southern perspective. Cambridge: Cambridge University Press, 1978. $455 \mathrm{p}$.

THORNTHWAITE, C.W. An appoach toward a rational classification. Geographical Review, New York, v.38, n.1, p.5594, 1948.

THORNTHWAITE, C.W.; MATHER, J.R. The water balance. Centerton: Drexel Institute of Technology - Laboratory of Climatology, 1955, 104 p. (Publications in Climatology, v.8, n.1)

VICENTE, A.K.; NUNES, L.H. Extreme precipitation events in Campinas, Brasil. Terrae, Campinas, v.1, n.1, p.60-62, 2004.

WILKS, D.S. Theoretical probability distributions. In: Statistical methods in the atmospheric sciences. 2.ed. San Diego: Academic Press, 2006. p.102-104. 